\title{
De l'agglutination à la triangulation ou comment expliquer certaines séries morphologiques
}

\author{
Lignon, Stéphanie ${ }^{1}$, Namer, Fiammetta ${ }^{1}, \&$ Villoing, Florence ${ }^{2}$ \\ ${ }^{1}$ UMR 7118 ATILF \& Université de Lorraine \\ ${ }^{2}$ UMR 7023 SFL \& Université Paris 8 \\ \{stephanie.lignon et fiammetta.namer\}@univ-lorraine.fr \\ villoing@u-paris10.fr
}

\section{Introduction}

Le français comprend des séries de lexèmes comportant des suites régulières de composants morphologiques adjoints à des bases lexicales : c'est ainsi que dans biologiste, on retrouve -logie et -iste, que parisianisme comporte les séquences -ien et -isme, que mathématicien contient -ique et -ien et brumisation, -iser et -ion. L'analyse de séries de ce type soulève de nombreuses questions et a déjà suscité l'intérêt de nombreux chercheurs.

Ainsi, Dal et Namer (2010) examine les noms de propriété ethnique, qui ont l'air, pour certains, dérivés à partir d'un adjectif ethnique (on retrouve américain dans américanité), lui même construit sur base toponymique (américain < Amérique) : la forme de ces noms de propriété fait apparaître une séquence (par exemple -anité) qui correspond à une suite de deux suffixes (-ain et -ité). Les auteurs étudient les raisons de la variation catégorielle des bases de ces noms de propriété : celle-ci est tantôt un adjectif, comme avec américanité, tantôt un toponyme, comme avec portugalité, directement dérivé de Portugal. Dans Koehl (2009), l'auteur se penche, quant à elle, sur les noms de propriété construits sur base adjectivale en -al ou -aire (mortalité, angularité), et du décalage observable entre la forme de ces noms (qui donnent à voir dans la séquence finale, -alité ou -arité, la succession de deux suffixes) et leur sens (qui se calcule par rapport au nom de base de l'adjectif: la mortalité désigne le nombre de morts, l'angularité la mesure d'un angle). Dans le même ordre d'idée, Namer (2013) analyse les verbes dont l'infinitif se termine par -aliser ou -ariser, dont la base se réalise sous la forme d'un radical adjectival dénominal et qui s'interprètent soit relativement à cet adjectif (populariser $=$ « rendre populaire »), soit relativement au nom de base de cet adjectif (instrumentaliser $=$ « transformer en instrument $»)$.

Enfin, Roché (2009) décrit des noms en -isation, -icien, -alité, -alisme, -arisme et -ianisme pour lesquels il formule une hypothèse constructionnelle fondée sur ce qu'il nomme une «transitivité de la motivation ». Ainsi, il démontre les insuffisances des analyses morphologiques en chaîne, pour lesquelles les formes examinées seraient exclusivement, et systématiquement, motivées par leur base immédiate, et donc le fruit de deux suffixations successives.

Les questions posées dans notre article et les résultats auxquels nous parvenons se situent dans la lignée des études ci-dessus, et font, plus précisément, suite au travail exposé dans Roché (2009).

Nous avons sélectionné trois types de noms: les noms en -icien (diététicien), en -ianisme (confucianisme), en -isation (benladenisation) et en -logiste (dermatologiste). Ces noms sont non seulement caractérisés par une apparente succession d'affixes (ou composants), i.e., respectivement, -ique et -ien, -ien et -isme, -iser et -ion, et -logie et -iste ou en -logue et -iste. Mais nous montrons que ces combinaisons d'affixes répondent à des motivations différentes en répondant aux questions ci-dessous : - Quelle est la nature de l'attraction entre les composants morphologiques qui constituent ces suites ?

- Ces suites sont-elles analysées/analysables comme un unique exposant ou une suite de deux exposants?

- La régularité de telles constructions est-elle l'indice de l'émergence de nouvelles règles ? 
Enfin, nous proposons pour les noms en -icien, -ianisme, -isation et -logiste un modèle d'analyse non linéaire, réalisé sous la forme d'une représentation ternaire, comme les auteurs ci-dessus l'ont fait avant nous. En outre, notre analyse fait apparaître des différences au sein même de ce modèle, en fonction des rapports existant dans les familles morphologiques des quatre types de noms étudiés.

Pour ce faire, nous étudierons, dans un premier temps, les noms en -ianisme et -icien dont la construction est motivée par des raisons phonologiques (§. 2), puis les noms en -logiste motivés par des raisons sémantiques (§. 3) et enfin les noms en -isation motivés discursivement (§. 4). La dernière partie (§. 5) tentera une analyse unifiée de ces formations.

\section{Motivation phonologique : le cas des noms en -ianisme et en -icien}

De nombreux travaux ont montré l'influence de paramètres phonologiques sur la construction morphologique. Pour le français, Plénat (1996a, 1996b, 1996c, 1997a, 1997b, 2000), et à sa suite Roché (1997, 2003a, 2011a) et Lignon (1999, 2000, 2013), entre autres, ont montré à de nombreuses reprises l'existence d'une contrainte dissimilative, visant à empêcher la consécution de phonèmes identiques ou proches lors de la construction d'un nouveau lexème, selon un modèle adapté de la théorie de l'optimalité (Prince \& Smolensky, 1993 ; Mc Carthy \& Prince, 1993). Les stratégies d'évitement sont diverses : soit la base peut être réparée : par troncation, épenthèse (Plénat \& Roché, 2012) interfixation (Roché, 2002, 2003b, 2005 ; Plénat \& Roché, 2004 ; Plénat, 2005), ou utilisation d'un allomorphe (Boyé \& Plénat, à paraître), soit le locuteur a recours a des solutions plus radicales. Dans ce dernier cas, il peut soit utiliser une autre forme appartenant à la famille dérivationnelle du lexème base (Roché 2008, 2009, 2011a), soit à un affixe concurrent, sémantiquement moins bien adapté, mais n'enfreignant pas la contrainte dissimilative (Roché, 1997 ; Plénat, 1999 ; Lignon et Plénat, 2011). Les noms en -ianisme et en -icien semblent être la résultante de l'impact de contraintes dissimilatives. Pour ce qui est des formes en -icien, leur apparition s'explique par le blocage du suffixe attendu. Il s'agit donc d'une substitution affixale, alors que l'apparition de formes en -ianisme est due à la sélection d'un radical favorable au suffixe attendu. Dans les deux cas, on effectue une réparation de la base, mais en adoptant des stratégies différentes : dans l'un des cas, échangisme suffixal et, dans l'autre, sélection de la forme adjectivale comme radical utilisé par l'opération constructionnelle.

\subsection{Les formes en Xisme versus Xianisme}

Dans l'article consacré au suffixe -isme, le $\mathrm{TLF}^{1}$ explique la forme messianisme, en relation morphologique avec messie, par un élargissement du suffixe -isme mais, dans la partie Morphologie, il constate que ce suffixe s'accole à d'autres suffixes, notamment à -ien comme par exemple dans les cas de cartésianisme < cartésien (de Descartes), hégélianisme ${ }^{2}<$ hégélien (de Hegel), etc. S'il est vrai que le substantif messie ne dispose pas d'un adjectif associé en -ien, ces différents cas semblent trop proches pour ne pas au moins s'interroger sur leurs similitudes. Roché $(2007,2009,2011 \mathrm{~b})$ observe que la suffixation en -isme sélectionne des formes adjectivales par ce qu'il appelle la «substitution paradigmatique » (2011: 83), c'est-à-dire que, lorsque -isme sélectionne les bases adjectivales, cette sélection n'est souvent que formelle. En effet, dans les cas d'adjectifs relationnels, l'interprétation de la forme en -isme se fait à partir du nom et non de l'adjectif: le colonialisme prône le maintien et le développement des colonies tout comme l'institutionnalisme est une "doctrine économique qui met l'accent sur le rôle joué par les institutions dans l'activité économique » (TLFi).

Cette substitution paradigmatique, caractérisée par la sélection de la forme adjectivale de la base, est quasi systématique avec les adjectifs construits en -al ou en -aire. Mais dans les cas d'adjectifs présentant une finale susceptible de se réaliser sous la forme d'une sifflante, comme par exemple, les adjectifs construits en -ique ou en -eux, se réalisant/is/ et /oz/ devant suffixe, le problème est plus complexe (cf. Roché, 2009). Pour les adjectifs en -eux, il est difficile de dire si le construit en -isme l'est à partir de la forme adjectivale suivie d'une tronction de la dernière syllabe ou de la forme nominale, ces deux 
séquences étant identiques : moyen âg(eux) / Moyen Âge. L'objectif est de toutes façons de respecter ainsi la contrainte de dissimilation (Grammont, 1895 ; Plénat, 2011). Lorsque la base est suffixament longue, les adjectifs en -ique construiront leurs dérivés en -isme par haplologie puisque la finale -ique se réalise /is/ devant suffixe. Ainsi, par exemple, nautique donnera nautisme au lieu de nauticisme. Quand l'adjectif et le nom disposent d'une forme identique, il peut parfois être difficile d'identifier la forme ayant servi à construire le dérivé (par exemple: botanique , $_{\text {botanique }}$ et botanisme). Des cas tels que celui d'asiatisme permettent de lever cette ambigüité. En effet, l'asiatisme peut s'interpréter comme une « idéologie favorable à l'Extrême Orient » (TLFi), donc à l'Asie. La forme asiatisme est le résultat de la sélection de l'adjectif asiatique, après haplologie. On pourrait penser que seule une volonté dissimilative a motivé la sélection de l'adjectif asiatique comme base d'asiatisme. Asie, monosyllabe dont la dernière attaque est une sifflante, se prête effectivement mal à la suffixation en -isme. La sélection de la forme de l'adjectif, asiatique, permet alors de construire, par haplologie, une forme n'enfreignant pas la contrainte dissimilative : asiatisme au lieu du possible asisme. Mais la sélection de la forme de l'adjectif en -ique n'est en rien exceptionnelle. En effet, les formes anacréontisme, angélisme, aristotélisme (au lieu des possibles anacréonisme, angisme, aristotisme) montrent qu'il s'agit bien d'une haplologie à partir des adjectifs en -ique (anacréontique, angélique, aristotélique) plutôt qu'une construction à partir des formes nominales (Anacréon, ange et Aristote), qu'aucune contrainte dissimilative ne motivait. La suffixation en -isme semble donc bien sélectionner la forme adjectivale lorsque celle-ci existe.

Les exceptions (pilosisme, scolasticisme) s'expliquent pour la première par la taille de la base : pileux est dissyllabique et ne peut être tronqué, puisqu'on obtiendrait ainsi une forme dans laquelle le radical de la base serait monosyllabique et ne répondrait donc pas au format minimal dissyllabique attendu des radicaux (pilisme) comme l'ont remarqué Plénat et Roché (2003) et Roché (2007, 2011b). Pour le second cas, scolasticisme, la troncation de la syllabe incriminée ne règlerait pas le problème, puisque la coda de la syllabe pénultième est également une sifflante (scolastisme).

Il semble donc que la suffixation en -isme sélectionne la forme de l'adjectif plutôt que celle du nom (autoritaire $>$ autoritarisme, en l'aménageant éventuellement lorsque sa forme présente une finale incompatible avec l'adjonction de la séquence /ism/ : romantique > romantisme). En revanche, les noms en relation morphologique avec des d'adjectifs en -ien présentent des dérivés en -isme soit construits sur la forme nominale (giscardisme, mendélisme, pirandellisme, apparemment construits sur Giscard, Mendel et Pirandello), soit sur la forme adjectivale (parisianisme, hégélianisme, luciférianisme, apparemment construits sur parisien, hégélien et luciférien). La sélection de la forme du radical semble plus aléatoire pour les construits en -ien que pour les autres formes adjectivales.

\subsubsection{Les Xianisme}

Roché $(2007,2011 b)$ et à sa suite Plénat (2011) expliquent l'existence de formes en -ianisme (désormais Xianisme) par la présence d'une contrainte dissimilative, qui bloquerait l'adjonction de -isme à des bases présentant en finale ou en dernière attaque une sifflante : Paris / parisien / parisianisme au lieu du possible parisisme. En effet, sur les 905 formes en -isme présentes dans le TLFi (une fois ôtées les formes en -ianisme), seules 67 formes ont une attaque devant -isme en sifflante (désormais XSisme): catholicisme, narcissisme. Sur les 40 formes en -ianisme, 17 présentent des sifflantes devant -ianisme (ou en coda de la syllabe pénultième) (désormais XSianisme) : keynesianisme, ossianisme. La répartition des attaques en sifflante est donc très différente : $42,5 \%$ des Xianisme présentent une attaque en sifflante contre environ $7 \%$ dans les Xisme, ce qui semble montrer que l'existence de formes en Xianisme est sans doute au moins partiellement motivée par la présence d'une sifflante en attaque devant -ien.

Pour toutes les formes en XSianisme, $\mathrm{X}$ a une taille inférieure ou égale à deux syllabes. De nombreux travaux ont montré que la taille minimale de la base doit être dissyllabique. Il est donc impossible de tronquer X afin de se débarrasser de la syllabe incriminée : la suffixation en -isme appliquée à Malthus ne pouvait donner autre chose que malthusianisme, malthisme disposant d'un radical monosyllabique et malthusisme enfreignant la contrainte dissimilative. Les seules exceptions sont les formes rosicrucianisme et confucianisme pour laquelle $\mathrm{X}$ est trissyllabique. Toutefois, pour rosicrucianisme, la 
base est un nom composé de deux monosyllabes (Rose Croix). Il est fort probable que seule la deuxième partie du composé soit prise en considération dans le compte des syllabes. Rosicrucianisme est donc interprété comme construit sur une base monosyllabique. Pour confucianisme, la troncation de la syllabe finale n'aurait conduit qu'à produire la forme confucisme, ce qui ne réglait en rien le problème de dissimilation.

\subsubsection{Les Xianime ne présentant pas de sifflantes}

Un problème demeure : si l'existence des formes en -ianisme est justifiée par le respect d'une contrainte phonologique, pourquoi construire des formes en -ianisme dans les cas où la base ne présente pas d'incompatibilité phonologique avec -isme? Les 23 Xianisme restants s'expliquent pour certains d'entre eux par le fait qu'ils sont vraisemblablement des emprunts à leur langue d'origine : baconianisme et zwinglianisme sont sans doute des calques de l'anglais baconianism et zwinglianism et hégélianisme de l'allemand hegelianismus. D'autres ne sont pas issus d'adjectifs construits en -ien: le novatianisme est la doctrine de l'anti-pape Novatien et le Fénianisme, la doctrine des Fénians. Certains encore sont d'un emploi rare et/ou doublonnent une forme existante en Xisme: augustinianisme, newtonianisme. Victorianisme se justifie car il renvoie à l'époque victorienne et non à la reine Victoria. Il ne s'agit nullement d'un cas de substitution paradigmatique. Enfin, nous reviendrons sur le cas de marianisme (section 2.1.4).

Les cas de Xianisme ne présentant pas de sifflante avant -ien sont finalement sans doute bien inférieurs à ce que les chiffres laissent penser.

\subsubsection{Les XSisme}

Les sections précédentes ont montré que la suffixation en -isme était sensible à la dissimilation et tentait d'éviter la consécution de deux sifflantes dans la même syllabe. On s'attend donc à ne pas trouver de formes présentant une sifflante devant -isme (désormais XSisme). Or le TLFi en enregistre 67 (eg. belgicisme, dreyfusisme). On a vu qu'il existait au moins deux moyens d'aménagement de ce type de bases : la troncation (eg. les adjectifs en -ique et en -eux) ou la substitution paradigmatique (eg. les adjectifs en -ien).

Pour ce qui est de l'hypothèse de l'aménagement par troncation, sur les 67 formes, 51 ont une base analysable comme étant inférieure à trois syllabes (eg. névrose/névrosisme, obtus/obtusisme, public/publicisme, etc.). Ces formes semblent donc difficilement tronquables. L'autre solution de réparation serait d'utiliser un radical existant dans le paradigme dérivationnel, comme la forme adjectivale. Seules cinq d'entre elles ont une forme en -ien dans leur famille morphologique (au sens de Hathout, 2011) : balzacien / balzacisme, dreyfusien / dreyfusisme, maurrassien / maurrassisme, stö̈cien / stoïcisme, technicien / technicisme, pour lesquelles on aurait pu attendre une construction à partir de la forme adjectivale. Mais une telle sélection est problématique au moins pour balzacianisme et maurrassianisme, car si cela permet d'éviter la consécution de sifflantes, cela entraîne la consécution de /A/, balzacianisme et maurassianisme. Balzacisme est donc la moins mauvaise forme, bien qu'elle ne soit pas totalement satisfaisante. On trouve 162 pages indexées par Google sur la Toile pour balzacianisme contre 84000 pour balzacisme. La même requête effectuée à partir d'un anthroponyme dont la notoriété sur la Toile est légèrement inférieure (Balzac 3230 000/ Flaubert 1940 000) montre que l'utilisation d'une forme ne posant pas de problème a une utilisation plus fréquente (Flaubertisme: 27400 / Flaubertianisme 2). La forme dysphonique est visiblement évitée.

Restent 16 formes dont le radical a une taille supérieure à deux syllabes (eg. catholicisme, molinosisme). Parmi ces 16 formes :

- Deux ont une valeur oppositive : esthéticisme par rapport à esthétisme et organicisme par rapport à organisme. Le TLF propose la définition suivante pour esthéticisme « Théorie selon laquelle toute expression artistique et toute appréhension du sentiment du beau seraient plus affaire de 
sensibilité que de raison.» dont on voit bien la différence avec l'esthétisme. Quant à l'organicisme, il s'agit d'une doctrine : on est donc bien loin de l'organisme ;

- Une a une sifflante dans la syllabe pénultième, donc une troncation n'aurait pas réglé le problème : scolasticisme (/skolastisism/) ;

- Deux semblent empruntées construites : agnosticisme à l'anglais agnostism, et anatocisme au latin anatocismus ;

- Trois sont des doublets peu usités d'autres formes en Xisme: platonisme / platonicisme, romanticisme / romantisme, rosicrucianisme / rosicrucisme ;

- Spinoza, bien que trissylabique, ne fait plus que deux syllabes après troncation afin de construire spinozisme ;

- Quelle que soit l'analyse de la construction que l'ont fait de narcissime (construit sur Narcisse ou narcissique), il n'y avait pas de bonne solution et la construction d'un dérivé en -isme devait fatalement entraîner la réalisation d'une forme dysphonique.

Là encore, les XSisme construits à partir d'une base supérieure à deux syllabes sont sans doute moins nombreux que les chiffres ne le laissent présager.

\subsubsection{L'effet de série}

Un effet de série, déjà remarqué chez Roché (2011b), est imputé selon lui à un leader word, christianisme (2007 : 121), poursuivi par la série des anthroponymes générant des Xianisme. Le cas de messianisme, cité par le TLF comme cas d'élargissement du suffixe (cf. supra §1), est de ce point de vue tout à fait intéressant. En effet, messianisme appartient au même champ référentiel que christianisme, mais contrairement à ce dernier, il ne dispose pas d'un adjectif en -ien associé. La forme messianisme au lieu du possible messisme ne peut s'expliquer que par la volonté de résoudre une contrainte dissimilative en calquant le modèle de christianisme (et pas par une substitution paradigmatique, puisqu'il n'existe pas d'adjectif messien). Cet effet de série a pu, de surcroît, se trouver renforcé par une série d'emprunts à l'anglais, certains anciens (presbytérianisme, luthér(i)anisme, de l'anglais presbyterianism et lutherianism) et d'autres visiblement plus récents (sproutarianisme (ang. sproutarianism, de sprout, 'germe, pousse'), endianisme (ang. endianism, de end, 'fin, bout'), tractarianisme (ang. tractarianism, du « Tractarian Movement »).

(1) Hier, en navigant sur Wikipédia, je suis tombée sur le mot «sproutarianisme », qui désigne un régime végétarien basé sur la consommation de graines germées ${ }^{3}$.

(2) Endianisme : manière de ranger les octets en mémoire.

(3) Il a abandonné la religion Anglicane, poussé par le Tractarianisme, également connu sous le nom de mouvement d'Oxford et il devint un prêtre Jésuite.

La pression lexicale est telle que l'on va pouvoir utiliser cette séquence en -ianisme afin de construire des noms de religions relativement improbables. Ainsi, les fervents adeptes de la technologie vénéreront le geekianisme (à partir de geek, que l'on peut définir comme personne adepte des nouvelles technologies). Toutes les occurrences rencontrées sur la Toile placent geekianisme dans un contexte où il est nécessairement interprété comme nom de religion et pas simplement courant de pensée.

(4) oui, j'ai une religion : le geekianisme ${ }^{\wedge}$ oui, et je trouve qua sa a pas de gout particulier, ni bon ni mauvais.

(5) Je suis un fervent croyant du Geekianisme Rolling Eyes.

(6) En voyant ça je me demande ce qui fait le plus de dégâts, le christianisme ou legeekianisme. 
Bien que ces formes soient très marginales, elles sont bien la preuve de l'existence d'un modèle de construction de noms de religion à l'aide de la séquence -ianisme et sans avoir nécessairement besoin d'une base en Xien.

On rencontre également d'autres formes, encore plus surprenantes, comme marianisme, défini par le TLF comme la «tendance à accorder dans la spiritualité une place prévalente, parfois excessive à la Vierge Marie », alors que l'adjectif relationnel lié à (la Vierge) Marie est marial'. Si l'on en croit une requête effectuée sur la Toile à l'aide du moteur de recherche Google, le mouvement favorable au plagiat n'est pas le plagisme (sans doute à cause d'une possible ambigüité avec une base plage), mais le plagiarisme (forme construite sur le radical plagiaire, sans doute par attraction avec les construits à partir de la forme des adjectifs en -aire). Le plagiatisme, construit directement à partir du nom plagiat, arrive en deuxième position et plus surprenamment, en troisième position, le plagianisme, alors même qu'il n'existe pas d'adjectif en -ien construit sur plagiat. Ces deux formes, plagianisme et marianisme, ne peuvent s'expliquer que par l'impact de la série des Xianisme et l'attirance générée par la finale en /A/ des formes marial et plagiat (ou plagiaire) avec l'allomorphe savant de -ien /jan/ au sens de Dell et Selkirk (1978) qui se trouve à l'initiale de la séquence-ianisme.

(7) Il étudie ce qu'il appelle le plagianisme et le définit ainsi 128 : « Le plagianisme des orateurs est l'art ou la manière ingénieuse et facile, dont les auteurs plagianistes se servent adroitement et heureusement, pour changer ou déguiser toute sortes de discours ... »

L'asianisme est également un cas remarquable. Il présente une finale en -ianisme tout à fait inattendue dans la mesure où Asie ne comporte pas de dérivé en -ien dans sa famille constructionnelle. Le choix de la forme en -ianisme (8) permet de respecter la contrainte dissimilative, mais aussi de construire une forme sémantiquement distincte de l'asiatisme (9) tout en s'insérant dans la série des Xianisme :

(8) La rhétorique classique désigne par asianisme (c'est-à-dire style d'Asie mineure) un style de discours mis au point au iiie siècle av. J.-C. dans les villes grecques d'Ionie.

(9) Asiatisme (TLFi) : A. Caractère particulier rappelant un des traits dominants des Asiatiques. B. Idéologie favorable à l'Extrême-Orient

La pression de la série est telle qu'on trouve également des noms de doctrines émanant d'hommes politiques, pour lesquels l'adjectif relationnel usuel n'est pas en -ien (hollandais, sarkoz(y/i)ste et lepéniste) et pour lesquels il existe déjà une forme en -isme (hollandisme, sarkoz(y/i)sme, lepénisme) :

(10) L'hollandianisme sera encore pire lui qui continuera le sarkozysme de la manière la plus abjecte : en pillant le peuple et en dépeçant la France

(11) exemple, question test : que pense Jean-François Copé du "sarkozianisme"?

(12) ... obligatoirement connaître les systèmes de Management à la pointe des Etats comme le sarkozisme, cauhuzianisme, melanchonnisme et le lepenianisme.

Bien que très marginales, ces formes montrent bien l'existence, chez le locuteur, d'un modèle de construction de dérivés en -ianisme afin de construire des noms de doctrines (les dérivés axiologiques chez Roché, 2011b)

On pourrait penser que la série des $\mathrm{X} / \mathrm{Xien} / \mathrm{Xianisme} \mathrm{comporte} \mathrm{des} \mathrm{formes} \mathrm{liées} \mathrm{entre} \mathrm{elles} \mathrm{par} \mathrm{une}$ relation morphologique linaire orientée :

$\begin{array}{llllll}\text { (13) } X & \rightarrow & \text { Xien } & \rightarrow & \text { Xianisme } \\ \text { (14) Prusse } & & \rightarrow & \text { prussien } & \rightarrow & \text { prussianisme }\end{array}$

Or l'analyse des données montre que le Xianisme n'est pas nécessairement en lien avec le Xien, mais peut être interprété à partir du X et, inversement, qu'il est possible de générer un Xianisme sans besoin de la forme en Xien (cf. exemples (4) à (9)). Cette analyse sera développée dans la section 5. 


\subsection{Les $X(t)$ icien}

\subsubsection{Les données}

Il a déjà été remarqué l'attirance de la suffixation en -ien pour les bases en -ique (Nyrop, 1936 ; TLF ; Lignon et Plénat, 2009): mathématiques / mathématicien, domotique / domoticien, etc. au lieu des possibles mathématiciste et domoticiste. Lignon et Plénat (2009) montrent que cette attirance est explicable à l'aide de paramètres exclusivement morphophonologiques. Lorsque les bases présentent une sifflante en finale (ou en dernière attaque), elles sont défavorables à l'adjonction de cet affixe: mathématique, qui se réalise /matematis/, est peu adapté à la suffixation en -iste. Le suffixe -ien vient donc prendre le relais dans les cas où aucun autre mode de réparation n'est possible : bases dont la taille est inférieure à trois syllabes ou bases en -tique : musique / musicien, tactique / tacticien (pour plus de détails sur cette analyse, cf. Lignon et Plénat, 2009). Le contexte phonologique est extrêmement contraint: -ien n'intervient pour construire des noms de spécialistes que dans les cas de bases en sifflantes et essentiellement des bases à finale -ique qui se réalisent /is/ devant suffixe. Cette attirance quasi exclusive de -ien pour les bases en -(t)ique afin de construire des noms de spécialistes entraîne l'apparition d'une finale -icien, associée aux noms de spécialistes. Les locuteurs analysent alors les formes telles que organicien comme construites soit sur organique avec le suffixe -ien, soit sur organe, avec le suffixe -icien.

\subsubsection{Le cas des formes en -icien non enregistrées dans les dictionnaires}

La séquence en -icien est donc identifiée par les locuteurs comme susceptible de construire des noms de spécialistes et associée à des noms de disciplines en -ique. De fait, on rencontre sur la Toile des construits en -icien et en -ique non enregistrés dans le dictionnaire, où la forme en -icien renvoie à un spécialiste d'une discipline, d'un concept, d'une pratique et où la forme en -ique attribue le statut de discipline «théorique », de science à une chose qui, jusque là, ne semblait pas nécessiter ce qualificatif. La finale -ique apporte un aspect plus sérieux, plus professionnel, en associant à la base l'idée de science ou de théorie.

C'est ainsi que l'on parle désormais de qualiticien, spécialistes de qualitique, pour des personnes dont le rôle est de se préoccuper de la qualité des produits.

(15) La qualité est l'objet d'une démarche socio-économique que l'on appelle souvent qualitisme, laquelle relève d'une science - au moins d'une technique - dite qualitique.

S'il existe une forme en -(t)ique dans le paradigme dérivationnel, même si elle n'est pas a priori nominale, le spécialiste sera dénommé par une forme en - $(t)$ icien. Le spécialiste des médias est ainsi un médiaticien et tout aussi logiquement, la médiatique sera la science des médias :

(16) Puis, le médiaticien surveille de près la tournure prise par les événements

(17) La médiatique - Le traitement publicitaire des médias: Amazon.fr: Tarrit (Jean-Marc) et Tubik (Emmanuel): Livres.

Parfois, la forme en -ique peut également remplacer une forme en -ing. Le markéticien fera de la markétique, au lieu du markéting et/ou de la mercatique.

(18) J'avais l'habitude d'utiliser le mot markétique en lieu et place de l'affreux marketing qui n'a rien de français. Or on vient de me faire remarquer que ce mot n'existe pas !

(19) ... un petit appareil mécanique, robuste, swiss made pour de vrai, universellement copié sans être égalé - un vrai fantasme de markéticien aux bras noueux.

(20) La personnalité semble, en effet, émerger de toutes les conceptions différentes du markéticien qui s'expriment autour de la table. 
Le lien très étroit et exclusif entre formes en - $(t)$ ique et formes en -icien fait que l'apparition d'une forme en -icien entraîne dans son sillage une forme en -ique et inversement.

Mais la pression de ces formes va même au-delà. Les nouvelles technologies et les nouveaux médias ne sont pas avares de néologismes. On rencontre ainsi des wikiciens, alors même qu'il semble tout aussi peu probable, pour des raisons dissimilatives évidentes, que la forme wikique (science de l'utilisation ou de la création des Wikis) existe, tout comme une forme telle que l'ornithorynquique (science spécialisée dans l'étude des ornithorynques). Si on trouve une occurrence de ornithorynquique dans un emploi adjectival, c'est sans doute que cette forme dysphonique convient malgré tout à la dénotation de cet animal étrange. En revanche, les 30 pages recensées par Google pour la forme wikique ne comportent que des erreurs. Sur la Toile, on trouve également des facebookiciens, amateurs de réseaux sociaux qui préfèrent Facebook. Ceux qui utilisent Twitter sont, en toute logique, des twittericien, alors même qu'il ne s'agit pas de spécialistes mais d'utilisateurs et qu'il n'existe (pour l'instant) pas de facebookique ou de twitterique, comme théories de chacun de ces réseaux sociaux. Et ceux-ci seront sans doute étudiés par les geekiciens

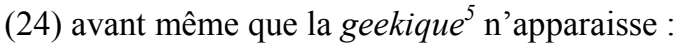

(21) Signez cette twitition si vous aussi vous en avez marre des facebookiciens sur twitter.

(22) 1 mai 2013 - Et quand vous faites \#SachezLe c'est une expression twittericienne?

(23) Chers Wikiciennes et Wikiciens! Pensez-vous que ces votations auront une influence sur MITIC ou seront-elles simplement mythiques?

(24) Bonjour les geekiciens, vous geekez sur quoi aujourd'hui ?

Il n'est alors plus besoin du support de la forme en -ique afin de créer un nom de spécialiste en -icien.

(25) Je l'ai piqué sur une page utilisateur d'un autre wikipédicien/ne.

(26) Il est softiciel, il fait des moteurs de recherche web avancés, des piscines et des home cinéma. softicien, c'est mieux.

Les formes en -icien donnent à voir deux choses : la première est qu'elles sont facilement associées à des formes en -ique, ce qui entraîne l'apparition quasi simultanée de couples Xique/Xicien, où Xique est le nom donné à la théorie et Xicien au spécialiste, alors même que l'existence d'un Xique n'était pas indispensable. La seconde, est que, malgré ce lien étroit, il semble que les formes en -icien prennent leur autonomie au regard des formes en -(t)ique et sont désormais utilisées afin de construire des noms de spécialistes ou d'utilisateurs.

\subsection{Bilan}

Les deux types de constructions étudiées dans les sections 2.1. et 2.2. s'expliquent grâce au respect de la contrainte dissimilative. Les moyens mis en œuvre afin de respecter cette contrainte sont différents. Dans un cas (les Xianisme), on opère la réparation au niveau du radical : le choix est fait de sélectionner la forme adjectivale afin d'éloigner le phonème problématique (ici, une sifflante) de l'exposant attendu (-isme). Dans l'autre (les Xicien), on fait le choix d'utiliser un autre exposant ne posant pas les mêmes difficultés phonologiques (-ien au lieu de -iste après les bases en sifflantes).

\section{Motivation sémantique : le cas des noms en -logiste}

Avec les noms se terminant par -logiste (biologiste, hématologiste), nous abordons une autre situation pouvant expliquer l'émergence de nouvelles séquences suffixales. Nous allons voir que l'étude des noms en -logiste et en -logue constitue une illustration de ce qu'on peut qualifier de " transfert de compétences » entre deux modes de formation de noms de spécialistes œuvrant dans des domaines scientifiques (et surtout médicaux). Les séquences -logue et -logiste s'observent dans des noms d'humains réalisant les schémas que nous noterons Xlogue et Xlogiste, et où X désigne un objet d'étude pour le spécialiste auquel réfère le nom construit ${ }^{6}$. 


\subsection{Les Xlogue et Xlogistes enregistrés dans les dictionnaires : synonymie}

\subsubsection{Les doublons Xlogue/Xlogiste}

\section{- Des synonymes absolus}

Le contenu d'un dictionnaire comme le TLF pourrait laisser croire que les noms de ces deux types renvoient exactement à la même classe de référents, ce que l'on constate dans les construits en -logue et -logiste qu'on y trouve, partageant la même séquence $\mathrm{X}$ et entretenant une relation de synonymie absolue au sens de Cruse (2004 : 154-157). 98 des 120 Xlogiste ont un doublet en Xlogue. Les noms en -logue constituent pour leur part un ensemble de 184 entrées de ce même dictionnaire.

(27) criminologue / criminologiste, ethnologue / ethnologiste, néphrologue / néphrologiste, zoologue / zoologiste

\section{- Des différences minimes}

Les différences entre les doublets sont minimes, et sont en rapport avec l'origine (les noms en -logiste résultent le plus souvent d'emprunts ou de calques de l'anglais), la fréquence et la date de première apparition de chaque forme. Ces différences font état de tendances qui varient pour chaque couple examiné :

- En étudiant l'évolution de l'utilisation des noms biologue et biologiste au moyen de Google Books Ngram Viewer ${ }^{7}$, on s'aperçoit que le premier nom est postérieur au second, dont l'apparition remonte au début du $19^{\text {ème }}$ siècle. On remarque également que la fréquence d'apparition de biologue a toujours été proche de zéro : ce nom n'a jamais été un concurrent sérieux à biologiste.

- Dermatologue et dermatologiste, selon la même source, apparaissent en même temps (vers 1830) et, jusqu'à récemment, le second est plus fréquemment employé que le premier. Cette tendance s'inverse définitivement en 1970, et dermatologiste est délaissé au profit de dermatologue.

- Toujours d'après Google Books Ngram Viewer, le nom anthropologiste apparaît aux environs de 1800, alors que les premières attestations de son concurrent anthropologue ne s'observent que 30 ans plus tard. La fréquence d'emploi du premier est supérieure à celle du second jusqu'en 1947, date à laquelle les courbes commencent à s'inverser. Un comportement analogue s'observe avec neurologue et neurologiste : le nom en -logiste est antérieur à la forme en -logue, qui devient hégémonique à partir des années 50 .

Comme on peut le voir, ni Xlogue ni Xlogiste ne l'emporte sur l'autre en matière d'antériorité ou de fréquence.

\subsubsection{Les Xlogue et Xlogiste isolés}

Les noms en -logue et en -logiste du TLF sans concurrent sélectionnent les mêmes types de base (des radicaux savants exploités dans la formation du lexique scientifique) et les noms d'humains produits sont spécialistes des mêmes domaines scientifiques et médicaux :

\section{(28) minéralogiste, psychogénéalogiste, néonatalogiste, climatologue, pneumologue}

Cette concurrence systématique entre les modes de formation en -logue et -logiste, que l'on constate d'après le contenu du lexique enregistré, semble être une anomalie linguistique : deux moyens distincts pour former des noms qui non seulement appartiennent au même type référentiel, mais qui de plus sont majoritairement des synonymes; cette situation aurait pu conduire à l'abandon total de l'un d'eux au profit de l'autre de la part des locuteurs. 


\subsection{Les Xlogue et Xlogistes de la Toile : disparité sémantique}

En réalité, l'examen des données extradictionnairiques montre qu'en synchronie, les deux schémas sont toujours disponibles; en revanche, seules les formes en -logiste continuent à dénommer exclusivement des spécialistes de disciplines rigoureusement scientifiques.

Une requête s'appliquant aux données de $\mathrm{frWaC}^{8}$ ramène 250 formes en -logiste et 714 en -logue néologiques relativement au contenu du TLF. Dans leur quasi-intégralité, les noms en -logiste désignent des savants, spécialistes d'une discipline médicale, scientifique ou littéraire (29). Dans de très rares exceptions, le terme est employé avec une intention humoristique (30) :

(29) gemmologiste, néonatalogiste, aquariologiste

(30) bayroulogiste (<François Bayrou: «Le Modem mourra de ses contradictions, car Bayrou est un Bayroulogiste centriste croyant, non-pratiquant. »), djangologiste (< Django Reinhardt : "Concert jazz: Le Trio Rosenberg et leurs amis Djangologistes à la Place des Arts »)

Loin de partager le caractère homogène des classes référentielles dénotées par Xlogiste, les noms en -logue renvoient, certes, eux aussi, à des spécialistes qui étudient une discipline scientifique, technique, (31a) ou non scientifique (31b), mais les locuteurs s'en servent également pour dénoter des individus dont le métier consiste à produire ce à quoi renvoie $\mathrm{X}$ (32), ou qui sont caractérisés par leur passion pour ce que désigne $\mathrm{X}(33)$.

(31) a. herpétologue ('spécialiste des reptiles et des amphibiens'), nivologue ('spécialiste de l'étude des caractéristiques de la neige et des couches neigeuses'), biérologue ('spécialiste de la bière').

(31) b. japonologue ('spécialiste du japon, de son histoire et de sa culture'), rugbyologue («Bons Matches, Allez les Petits, et bonnes 3èmes mi-temps. Jérôme Courcoux - rugbyologue pas officiel du tout »), licenciologue (consultant chargé de préparer les plans de licenciements).

(32) bruitologue ( Vocalchimistes, contrebarisseurs, bruitologues, ils utilisent leurs voix comme des instruments, les mots comme une musique et les sons comme un langage. », hymnologue (" Auteur d'une hymnologie »)

(33) tintinologue (< Tintin), nodologue ('spéc. des nœuds'), nirvanalogue ( $<$ Nirvana)

Tout individu voulant s'autoproclamer expert incontournable dans la connaissance de l'objet décrit par X se dépeint au moyen de Xlogue (34-35). Il en va de même pour toute personne propulsée au-devant de la scène médiatique en lien avec un événement social, politique, sanitaire, international, etc. mis en exergue par les professionnels de la communication. Ce sont ces mêmes professionnels qui popularisent ces nouveaux experts en inventant et diffusant ces nouveaux noms en Xlogue (36-37) :

(34) searchologue ( «Yahoo va devenir de plus en plus l'ami des geeks, du logiciel libre et des searchologues, en proposant des outils simples, utiles et fonctionnels »);

(35) sonologue («Quand j'ai dit qu’un critique de cinéma savait ce qu'étaient les images et les sons, évidemment je suis allée très vite, je n'ai jamais voulu dire que la culture d'un critique de cinéma était celle d'un iconologue ou d'un "sonologue ", si ça existe.) ;

(36) dahuologue ( « Ce grand Dahuologue expliqua ainsi que la durée de gestation de cet animal dépend du coté de la montagne où il se trouve, chacun sait que le dahu a soit les pattes gauches plus longues que les droites ou vice versa. »);

(37) polémologue ('géopoliticien spécialiste des conflits'), sondologue ('spécialiste des sondages'), préventologue ('en médecine, spécialiste de la prévention des risques et des accidents'). 
Plus remarquable encore, seules les formations en -logue peuvent servir de marqueurs diaphasiques : la désignation par Xlogue d'un spécialiste déjà défini au moyen d'un autre nom permet au scripteur d'accorder plus de prestige à la fonction de ce spécialiste (38), ou à l'inverse, la création d'un nom de spécialiste en -logue pour référer à une fonction jusque là inédite permet d'exprimer le mépris que le locuteur ressent pour l'individu, perçu comme un imposteur (39), ou la fonction, jugée vaine et prétentieuse (40). En marquant au moyen de Xlogue sa familiarité avec la fonction médicale de l'individu dénoté, le locuteur veut marquer sa proximité avec ce praticien, soit pour traduire un sentiment affectif, soit pour exorciser la crainte que lui inspire la relation patient/médecin dans laquelle il est engagé (41) :

(38) parturologue ('sage-femme'; < parturiente «(Femme) qui accouche; (femelle) qui met bas. »(TLFi)), radiatorologue ('garagiste' ; < radiateur), somnologue ('médecin traitant l'insomnie' ; < somnus 'sommeil')

(39) pédagologue («le pédagologue est celui qui parle de pédagogie sans la pratique »), négrologue («Cameroun: Stephen Smith, le Négrologue américain : le chef "Afrique" du quotidien français est l'auteur de l'article sur les escadrons de la mort »)

(40) bobologue («J'entends souvent dire, autour de moi, que le médecin généraliste est un docteur bobologue, comme si cela était dégradant d'être bobologue »), trucologue ( Ce qui permet aussi, aux toubibs, spécialistes et autres trucologues de cardiologie, de boyaulogie, d'exercer leur art majestueusement, pédamment »)

(41) bébélogue («Il n’a pas cessé de remuer à tel point que la "bébélogue" a eu du mal à prendre les mesures nécessaires. »), cervologue (« Il y a un passage qui m’a fait mourir de rire : celui sur une expérience pseudo-scientifique menée par un pschychiatrologiste cervologue cognito-alternatif!»), dingologue (« un "dingologue" pour vider ma tête me ferait le plus grand bien car à chacune de mes explosions je me sens vidée et bcp mieux »).

En conséquence, seuls les noms en -logue sont utilisés à des fins purement comiques, par la création d'énoncés où la succession de noms en Xlogue créés en rafale ont pour seul objectif la production de rimes (cf. Tanguy 2013) :

(42) «C'est quoi un pédagologue ?? -- Il répare les pédalos, qui naviguent sur nos beaux cours d'eau ?? -- Suis-je alors un cheminologue ?? -- Quitte à choisir, je préfère être un humourologue, mieux vaut le prendre comme ça. -- Si seulement je pouvais être éclairé par un éclairologue... »

\subsection{Xlogue et Xlogiste en lien avec Xlogie : disparité morphologique}

À partir de l'observation de ces données, Villoing \& Namer (à paraître) a montré que, contrairement à Xlogue (e.g. gynécologue), dont la formation est concommittante à celle du nom de domaine d'étude Xlogie (e.g. gynécologie), Xlogiste est dérivé en -iste de ce même Xlogie: l'existence du nom de l'humain spécialiste d'un domaine de spécialité est tributaire de l'attestation du nom dénotant ce domaine d'étude (sur -iste, voir Roché 2007, 2011b).

Cette analyse est appuyée par plusieurs arguments dont les principaux tiennent (i) d'une part à l'existence systématique de paires de noms en -logiste et -logie sans que soit obligatoirement impliqué un nom en -logue (à tout Xlogiste correspond un Xlogie cf. (43-45)) et (ii) d'autre part à l'absence de noms en -logiste si le nom en -logie n'est pas attesté (46-47), sachant que le nom en -logie peut exister indépendamment du nom en -logiste (48-51) :

(43) généalogie, généalogiste, généalogue

(44) xénobiologie, xénobiologiste, ${ }^{\circ}$ xénobiologue

(45) La xénobiologie est une sous-discipline naissante de la biologie synthétique qui vise la mise au point de formes de vie étrangères, du point de vue métabolique. 
(46) lunologue, ${ }^{\circ}$ lunologie, ${ }^{\circ}$ lunologiste (« lunologue : Spécialiste de la lune. TLFi) radinologue, ${ }^{\circ}$ radinologie, ${ }^{\circ}$ radinologiste (« et je suis radinologue de l'éclairage... »)

(48) chiracologue, chiracologie, ${ }^{\circ}$ chiracologiste

(49) «Et Chirac le décore de la Légion d'honneur dans son bureau de l'Élysée, signe qui ne trompe pas les « chiracologues »», « Aujourd'hui, cet expert en "chiracologie" sait que le président veut utiliser trois leviers dans la perspective de $2007 »$

$$
\text { pleurologue, pleurologie, }{ }^{\circ} \text { pleurologiste }
$$

«En résumé je préfère un rigologue à un pleurologue»/ «Effectivement! quand je pense qu'il y en a qui sont adeptes de la pleurologie (larmologie, etc.) »

En fait, la formation d'un nom en Xlogue et celle du nom en Xlogie partageant la même séquence X sont en relation de dépendance mutuelle, les deux noms se définissant par ailleurs par rapport à une base commune X.

\subsection{Bilan des contributions de Xlogue et Xlogiste et émergence d'un suffixe}

Pour le locuteur, la formation d'un Xlogiste répond à la nécessité d'ancrer sans équivoque celui qui est désigné par ce nom dans la classe référentielle des «véritables » spécialistes.

Certes, un spécialiste est aussi nommé par un nom en Xlogue, mais la polysémie de ce patron constructionnel (qui peut dénoter n'importe quel expert, ou collectionneur, ou amateur plus ou moins fanatique), affaiblit la perception que l'on se fait de son rôle de formateur de noms de spécialité.

Les témoignages d'internautes viennent confirmer cette analyse au point même que la séquence -logiste est perçue comme une unité à part entière, qui, face à -logue, garantit, aux noms qu'il construit, l'interprétation de spécialiste (cf. le commentaire (52) d'un internaute) :

(52) Logue semble désigner celui qui cause sur le sujet et logiste celui qui est très calé sur le sujet...

On voit en effet émerger dans les commentaires métalinguistiques d'internautes qui créent ou interprètent des mots en -logiste, la perception du fait que cette séquence serait un mode de formation unique, fruit de l'agglutination de deux suffixes, et dont la valeur sémantique est renforcée, puisqu'elle bénéficie du cumul des sens véhiculés par la suffixation en -iste d'une part, et la formation en -logue (53) ou -logie (54) d'autre part.

(53) En fait, le suffixe "logiste" est un suffixe agglutiné composé de deux suffixes distincts: logue + iste... dont le sens est essentiellement le même : ils désignent tous deux des personnes..

(54) Le suffixe «-logiste » est un suffixe agglutiné ; c'est-à-dire qu'il est composé de plusieurs suffixes ; c'est un suffixe complexe, en quelque sorte. Ce suffixe est le résultat de l'opération «-logie $»+\ll$-iste $»$

Ainsi, avec la série des noms en -logiste, nous assistons finalement à un double phénomène : d'une part, un mécanisme d'agglutination de deux formants : -logie et -iste, et d'autre part, ce qui peut être ressenti comme l'émergence d'un nouveau mode de formation en -logiste de la part de certains locuteurs. En tout état de cause, le rôle de cette séquence, qu'elle soit agglutinée ou néologique, est de renforcer, chez les référents des Xlogistes, l'impression de sérieux scientifique que doit dégager la classe des référents dénotés, grâce à l'effet combiné de deux marques, chacune étant par ailleurs identifiée comme relevant du champ sémantico-conceptuel de la connaissance, du savoir, et/ou de ses spécialistes. 


\section{Motivation discursive : le cas des noms en -isation}

Parmi les finales qui semblent trouver une sorte d'autonomie suffixale, on peut enfin mentionner le cas des formes en -isation (que nous noterons Xisation). Les lexèmes qui comportent cette séquence nominalisent des verbes suffixés en -iser (notés Xiser), et ce procédé de nominalisation sélectionne le radical en /izat/ du verbe, appelé radical savant (cf. Bonami \& al., 2009). Les noms dérivés désignent donc des actions ou leur résultat :

(55) homogénéisation : «action d'homogénéiser ou résultat de cette action »

Du point de vue de l'organisation du lexique construit, la construction du dérivé en -ion sur la base du verbe en verbe en -iser répond à un besoin discursif : la seule différence conceptuelle entre la base et le nom dérivé est celle qui est induite par le changement de catégorie grammaticale. C'est ainsi que les contenus sémantiques des énoncés (56a) et (56b) sont identiques :

(56) a. Homogénéiser l'échantillon de laboratoire

(56) b. L'homogénéisation de l'échantillon de laboratoire

Suivant Roché (2006), on considère que les noms en -ion résultent d'un processus de désignation de ce que dénote le verbe de base.

Sur la Toile, la requête 'isation' ramène quelques textes indiquant que, dans la perception qu'en ont leurs auteurs, cette séquence constitue un opérateur morphologique à part entière (57) :

(57) -ISATION / suff. Suffixe apportant la notion de "transformation"

Dans le même esprit, les exemples (58-62) trouvés en ligne comportent tous une forme en $X$-isation. La création de ces formes à l'aide d'un tiret indique que le scripteur y voit deux unités distinctes, la seconde marquant la transformation. C'est, en d'autres termes, par cette marque graphique qui la sépare de X que la séquence $<$ isation $>$ semble, aux yeux des internautes, constituer l'affixe unique construisant un nom de « transformation » (suivant la définition en (57)) conduisant à un état qui se définit par rapport au référent de X : ainsi, la garmin-isation fait référence à l'emploi de GPS de la marque Garmin, la logo-isation de l'espace désigne sa transformation en logos, la Wal-Mart-isation et l'Ikea-isation font référence, respectivement, à l'hégémonie des chaînes de magasins Wal Mart et Ikéa, et l'OMC-isation de l'ONU signale le processus de subordination de cet organisme à l' $O M C$ :

(58) garmin-isation - A tous ceux qui s'équipent d'un gps de marque garmin ou qui comptent s'en équiper, envoyez moi un petit message privé qu'on en parle

(59) l'espace public est devenu un symbole à part entière, faisant l'objet d'une véritable "logo-isation"

(60) Certains ont exprimé leur inquiétude face à la « Wal-Mart-isation »

(61) L'ONU en voie d'OMC-isation?

(62) L'« Ikea-isation » du monde - (Les Échos)

Un indice numérique peut être utilisé pour évaluer le degré d'indépendance que la formation des noms en -isation est en train d'acquérir vis-à-vis de celle des verbes apparentés en -iser : cet indicateur est fourni par des mesures de fréquence, calculées à partir des données lexicales contenues dans frWaC (voir section 3). Il repose sur trois constats, établis dans Dal \& Namer (à paraître), et les conclusions qu'en tirent les auteurs.

Le premier porte sur le nombre de formes distinctes construites respectivement en -iser et en -isation. Ces valeurs sont comparables (frWaC comporte 8948 verbes en -iser et 7253 noms en -isation) et pourraient faire penser qu'à $80 \%$ des verbes en -iser du corpus correspond une nominalisation en -ion, et que donc le procédé de formation des noms en -ion a saturé l'espace des bases verbales disponibles dans frWaC. 
Cette tentative d'analyse est cependant remise en cause par un second constat, qui nous apprend que, d'une part, $71 \%$ des verbes en -iser n'ont pas de nom dérivé et que, symétriquement, d'autre part, $65 \%$ des noms en -ion sont dépourvus de base verbale. Le premier chiffre contredit l'hypothèse d'une saturation des bases en -iser sélectionnables par le procédé de formation des noms en -ion, et rend compte dans le même temps de la productivité de ce procédé lorsqu'il s'applique aux bases verbales en -iser. Rappelons en effet que Dal et al. (2008) avait démontré qu'en général, le mode de formation de noms déverbaux en -ion est non productif. Les bases en -iser constituent une exception à ce constat. Le deuxième chiffre nous montre que, dans le frWaC du moins, la construction des noms en -isation peut se faire sans qu'il n'y ait trace du parent verbal. C'est ce qu'illustrent les exemples grégarisation et cabanisation cf (63a-b) ${ }^{9}$. Pour l'exemple (61), ainsi que les exemples sous (64a-c), extraits de la Toile, le verbe de base ${ }^{10}$ est, sinon non attesté, du moins très rare.

(63) a. L'objectif global de cette thèse est d'expliquer ce phénomène de grégarisation inédit à Madagascar.

b. Dans la région, $80 \%$ des communes littorales sont concernées par la cabanisation, qui bat son plein du côté de Salses-Leucate (Aude), de la Gardiole ou de l'étang de l'Or (Hérault).

(64) a. Personnalisation, ego-isation et désacralisation des rituels politiques.

b. ... le ringardisme, l'immobilisme, la NIAISERISATION? niais niaiserie niaiserisation, cherchez pas dans le dico, ce mot je viens de l'inventer.

c. C'est une tendance dangereuse vers l'intergouvernementalisation, vers l'onunisation de notre système de ressources

Ce premier résultat tend donc à montrer que la formation de nouveaux noms en -isation n'est pas tributaire de l'existence d'un verbe en -iser de la même famille morphologique, mais que, au contraire, le mécanisme d'activation de chaque procédé semble consubstanciel à celui de l'autre.

Une troisième mesure vient conforter cette hypothèse. Elle concerne les fréquences d'occurrences des formes nominales et verbales. Dans leur globalité, les noms sont presque deux fois plus fréquents que les verbes (2 288883 vs 1143 290). Pris individuellement, et si on tient compte uniquement des couples (Xiser, Xisation) dont les deux membres sont attestés, l'écart est au moins le même, ce qu'illustrent les exemples (65-67). Le nombre d'occurrences de chaque forme en -isation (65a, 66a, 67a) et -iser (65b, $66 \mathrm{~b}, 67 \mathrm{~b})$ est donné entre parenthèses ${ }^{11}$ :

(65) a. Rester vigilant face au phénomène «d'ONGisation » (107)

b. On aura beau les ONGiser à tour de bras pour leur proposer des solutions alternatives, ils préfèreront cent fois profiter de l'électricité nucléaire (20)

(66) a. C'est ce que l'auteur définit comme la « cocacolisation » (45)

b. Voilà de quoi montrer à nos enfants sans risque de les cocacoliser ou de les fastfoudiser un peu plus (9)

(67) a. Le contexte italien avait ouvert la voie à la berlusconisation (345)

b. Arnaud Montebourg, l'un des porte-parole de Ségolène Royal, a affirmé lundi que "la France est en train de voir se berlusconiser son système médiatique" (119)

L'examen du vaste échantillon de données que contient frWaC conduit à la conclusion suivante : la formation des Xisation est concomitante à celle des Xiser, en cela que les deux procédés de formation étudiés entretiennent une relation d'interdépendance, tout en ayant pour ascendant commun une forme $\mathrm{X}$ adjectivale ou nominale.

Xiser et Xisation entretiennent une relation non orientée, dont témoignent les mesures de fréquence commentées ci-dessus, car elles nous montrent une supériorité numérique (du nombre d'occurrences) des noms sur (celui) des verbes. Cet indice numérique conforte en effet l'hypothèse suivant laquelle -isation 
est de plus en plus perçu comme l'exposant d'un procédé à part entière, construisant des unités dont le contenu sémantique est identique à celui des unités verbales en -iser, à la fonction pragmatique près.

\section{$5 \quad$ Vers une analyse unifiée}

Le point de départ du travail présenté ici est l'étude de séquences apparemment construites par l'agglutination de composants morphologiques: -icien (-ique+-ien), -ianisme (-ien+-isme), -logiste (-logie+-iste) et -isation (-iser+-ion). L'étude que nous avons menée a permis de montrer que l'existence de ces séquences répondait à des besoins différents.

Ainsi, les cas de -icien et -ianisme s'expliquent à l'aide de motivations morphophonologiques, imputables au respect de la contrainte de dissimilation. Les stratégies pour y parvenir diffèrent: substitution paradigmatique pour-ianisme et substitution affixale pour-icien.

L'émergence de -isation en tant que nouveau patron constructionnel indépendant de l'attestation des verbes en -iser s'explique en partie par le registre textuel du discours journalistique dans lequel on rencontre les plus fortes concentrations de ce type de nominalisation : en effet, de nombreux travaux consacrés aux genres textuels ont montré, à la suite de Biber (1986), que les écrits journalistiques constituent un terrain privilégié pour l'emploi des noms déverbaux de procès.

Enfin, c'est une motivation sémantique qui conduit à la différentiation majeure que l'on observe depuis quelques décennies entre les formations en -logue et celles en -logiste : pour ces dernières, le segment identifié par les locuteurs renforce, chez les individus ainsi dénommés, leur qualité de spécialiste (et même d'hyperspécialiste).

Le constat que nous avons été amenées à faire est que, pour chacun de ces cas, la séquence s'autonomisait par rapport à l'analyse en chaîne qui aurait pu être proposée et que le scripteur peut reconnaître chacune de ces séquences comme l'exposant d'un (nouveau) procédé de construction de lexèmes.

Bien qu'ayant des causes dissemblables, l'émergence de ces nouveaux affixes (ou de ce qui est ressenti comme tel) est passible, comme nous allons le voir, d'une analyse similaire, qui se manifeste par une représentation ternaire de la relation entre les données impliquées dans chacun des mécanismes constructionnels.

Cette représentation se traduit par une structure triangulaire régie par trois principes :

(1) les arcs sont potentiellement non-orientés,

(2) les sommets peuvent être non instanciés,

(3) chacun des membres de la relation non-orientée peut être obtenu soit à partir de la connaissance de l'autre, soit à partir de leur ascendant commun.

Cette analyse unifiée est rendue possible par l'adoption d'un modèle qui constitue le prolongement du cadre théorique de la morphologie dite lexématique (pour un survol, voir Fradin, 2003 ; pour une application récente au français, voir Fradin et al., 2009). L'unité est le lexème, et le procédé de construction qui manipule cette unité se manifeste sous la forme d'une relation entre base(s) et construits où interagissent trois mécanismes simultanés et indépendants : l'un agit sur la forme, le deuxième établit le rapport catégoriel entre l'input et l'output et le troisième se charge de définir le lexème construit en fonction du sens de sa base. Par ce choix théorique, nous nous affranchissons des biais induits par les opérations de concaténation de morphèmes déclenchées ou bloquées suivant des contraintes pesant sur la base, et assignant aux mots construits un sens compositionnel, dont la complexité croît avec le nombre de constituants formels. Ces opérations sont caractéristiques des théories s'appuyant sur les courants morphématiques incrémentaux de la morphologie.

Nous allons voir comment cette représentation ternaire sert de révélateur des différences et des similitudes entre les quatre cas discutés aux $\S 2$ à 4 . En effet, l'ensemble des constructions étudiées est 
passible d'une analyse triangulaire. Toutes les constructions que nous avons étudiées manifestent les propriétés suivantes :

- elles mettent en jeu un schéma de lexèmes, que nous symbolisons par (X, Xm1, Xm2): m1 et $\mathrm{m} 2$ désignent des (suites de) marques de type affixe ou formant, et $X$ est l'ascendant morphologique de Xm1 et Xm2.

- $\quad \mathrm{Xm} 2$ est soit dérivé de $\mathrm{Xm} 1$, soit construit directement à partir de X.

Cependant, trois des structures étudiées se caractérisent par une relation morphologique non orientée qui ne s'observe pas avec la dernière (§. 5.1). Enfin, deux constructions obéissent au même modèle formel (§. 5.2), et deux partagent les mêmes rapports sémantiques (§. 5.3).

\section{1 (X, Xien, Xianisme) : un schéma orienté}

Les adjectifs en -ien (Xien) sont construits à partir d'une base nominale X. Ils peuvent ensuite être convertis en nom appartenant à des catégories référentielles différentes selon la nature sémantico-référentielle du $\mathrm{X}$ (habitant pour les $\mathrm{X}$ toponymes, spécialistes ou partisans pour les $\mathrm{X}$ anthroponymes, etc.). Nous avons vu au §. 2.1. que les formes en Xianisme sont analysables directement à partir de la base X (puisque la séquence -ien n'intervient que pour éloigner le -isme de la sifflante que comporte la base en finale ou en dernière attaque). Toutefois, ces Xianisme peuvent également s'interpréter comme construits directement sur l'adjectif en -ien dans la mesure où ce dernier étant purement relationnel, son emploi n'entraîne qu'une modification discursive, mais non sémantique, de l'emploi du nom base. Une forme telle que malthusisanisme pourra done s'analyser comme « ce qui est typique de Malthus » et/ou «ce qui est typique de (l'œuvre) malthusienne et/ou des malthusiens ». L'analyse obtenue est présentée dans la figure 1 ci-dessous. Les relations entre les trois sommets sont ici systématiquement orientées, et il est possible d'analyser les formes en Xianisme sans passer par la forme en Xien.

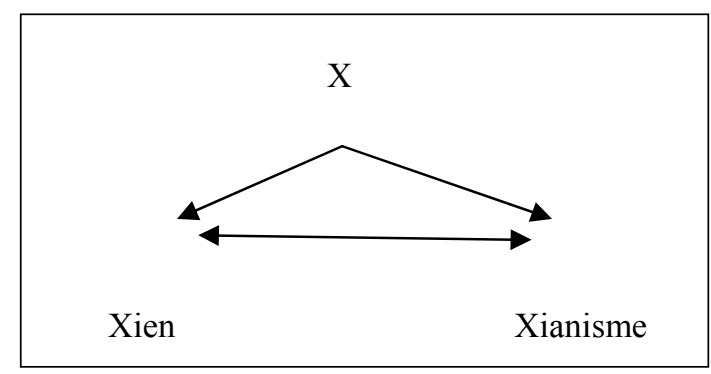

Figure 1 : Schéma ternaire (X, Xien, Xianisme)

Contrairement à la formation de Xianisme, celles de Xicien, Xisation et Xlogiste met en jeu une représentation partiellement non orientée.

\subsection{Xicien et Xisation : même modèle formel}

L'observation des Xicien et de Xisation nous amène à proposer un schéma identique pour les deux constructions et plus complexe que le précédent (fig. 1). Pour ce qui est des Xicien, s'il est possible de faire une analyse linéaire de leur construction en passant par la forme Xique, il est également possible de les construire directement à partir de X. Ainsi, le nom qualiticien s'interprète comme le 'spécialiste de la qualité ou 'de la qualitique'. Il est également possible de créer une forme en -icien sans nécessairement passer par Xique (cf. exemples (19)-(20)). Mais la relation entre Xique et Xicien n'est pas une relation orientée, ce qui implique que l'existence d'une forme en Xique conduit à la création d'un nom de 
spécialiste en Xicien et, inversement, qu'un nom de spécialiste en Xicien entraîne dans son sillage l'existence d'une spécialité en Xique. En conséquence, les deux formes (Xique et Xicien) peuvent se déduire l'une de l'autre.

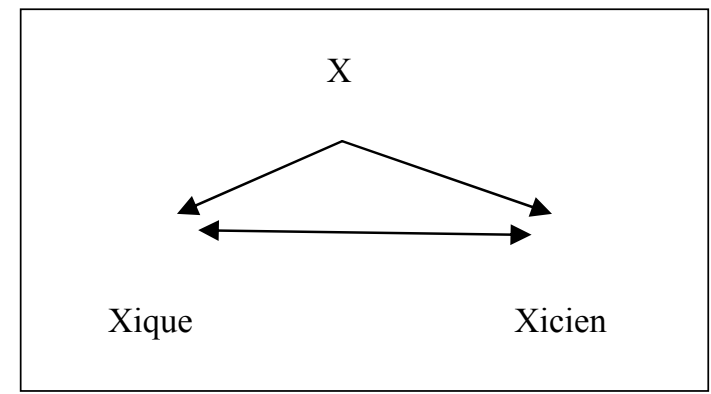

Figure 2 : Schéma ternaire (X, Xique, Xicien)

La formation des Xisation peut se voir d'une manière très similaire, et se représente sous la forme du schéma donné dans la figure 3, comme (Dal, 2004) l'avait déjà montré sur un nombre réduit de données.

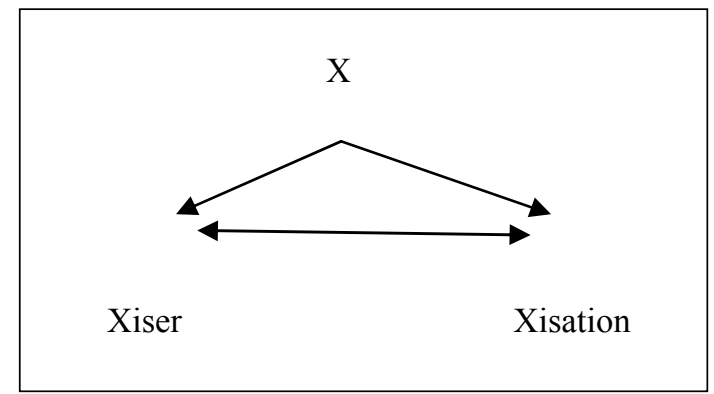

Figure 3 : Schéma ternaire (X, Xiser, Xisation)

Le parallélisme des figures 2 et 3 ne doit pas faire oublier que, si le mécanisme en oeuvre pour former Xicien et Xisation est le même, l'interprétation de l'axe bi-orienté est différente. En effet, Xisation et Xiser renvoient à la même catégorie sémantico-référentielle, i.e. celle des actions : les instances de ces deux types de lexèmes ont toujours un contenu sémantique très proche, alors que ce n'est pas le cas pour Xicien et Xique, qui appartiennent à deux catégories sémantico-référentielles bien distinctes, puisque Xicien appartient à la catégorie des noms de spécialistes alors que Xique fait partie de celle des noms de sciences ou de disciplines.

\subsection{Xicien et Xlogue : des mécanismes sémantiques similaires}

De fait, le lien sémantique qui s'établit entre Xicien et Xique rappelle celui que l'on observe entre Xlogue et Xlogie : Xicien et Xlogue désignent des spécialistes (ou qui se prétendent tels) de l'objet que dénote X, quand il existe, et ces spécialistes interviennent dans un domaine nommé, respectivement, par Xique et Xlogie : de même que le spécialiste et le domaine qui le concernent se justifient mutuellement, le nom du spécialiste et celui du domaine se définissent l'un par rapport à l'autre.

En effet, (X, Xlogue, Xlogie) réalisent la même structure partiellement non-orientée que l'on a dans la figure 2, et qui exprime le caractère interdépendant de la construction du nom du spécialiste et de celui de sa discipline.

Dans ce premier sous-système ternaire, chaque sommet du triangle résultant est potentiellement non attesté. C'est par exemple le cas du sommet potentiellement occupé par Xlogue (nous ne trouvons aucune 
occurrence de ultrasonologue, correspondant à $\mathrm{X}=$ ultrason et Xlogie ultrasonologie) et de celui occupé par Xlogie (entonoirologie ne semble pas exister, contrairement à entonoirologue, apparenté à entonoir). C'est enfin le cas de X, que le locuteur n'a pas besoin de connaître pour reconstruire le lien entre Xlogue et Xlogie (ainsi, il ne lui est pas nécessaire de comprendre que /ekin/ est le radical grec correspondant au nom oursin pour reconstruire échinologue à partir de échinologie, et inversement).

Finalement, on retrouve avec (Xlogie, Xlogue) le même caractère concommitant de la création du nom de l'expert avec celle du nom de la discipline que l'on observe aussi avec (Xique, Xicien), où énergéticien et énergétique par exemple peuvent se définir l'un l'autre, de même que, par exemple, climaticien et climatique. Xlogie et Xique se définissent soit par rapport à $\mathrm{X}$, soit, respectivement, relativement à Xlogue et Xicien. C'est ce que représente la figure 4.1 où se superposent en une seule structure $(\mathrm{X}, \mathrm{Xm} 1$, $\mathrm{Xm} 2$ ) les relations qu'entretiennent les membres des familles (X, Xlogue, Xlogie) et (X, Xicien, Xique). Comme l'indiquent les gloses, $\mathrm{Xm} 1$ et $\mathrm{Xm} 2$ se définissent chacun par rapport à l'autre, et tous les deux par rapport à X :

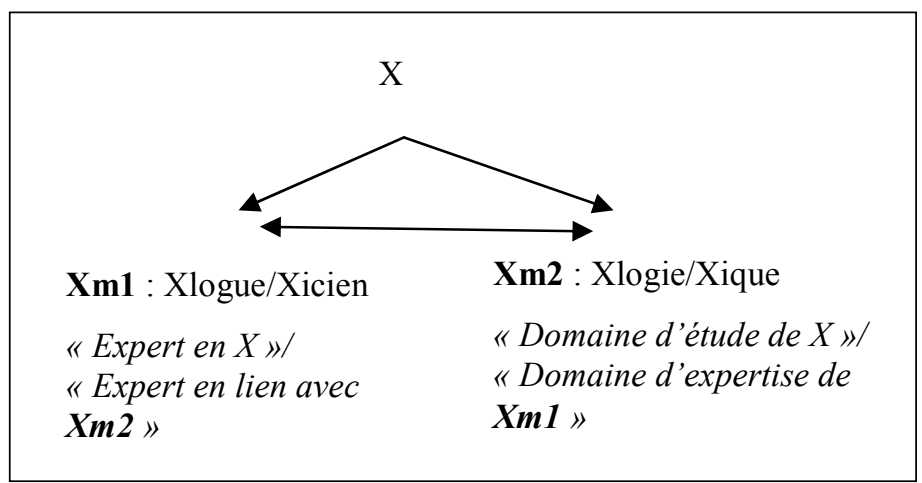

Figure 4.1 : Schémas (X, Xlogue, Xlogie) et (X, Xicien, Xique) et relations sémantiques

La différence entre ces deux familles morphologiques se manifeste avec la représentation de Xlogiste. Comme on le voit dans la figure 4.2, le mécanisme conduisant à leur construction complète la triangulation illustrée dans la figure 4.1: si Xlogie est non attesté, alors on ne trouve pas non plus de Xlogiste. Il resterait à imaginer comment on pourrait, dans ce schéma, représenter le fait que 'logiste' s'interprète, chez certains locuteurs, comme un suffixe à part entière, si l'on en croit les témoignages reproduits en (52-54)).

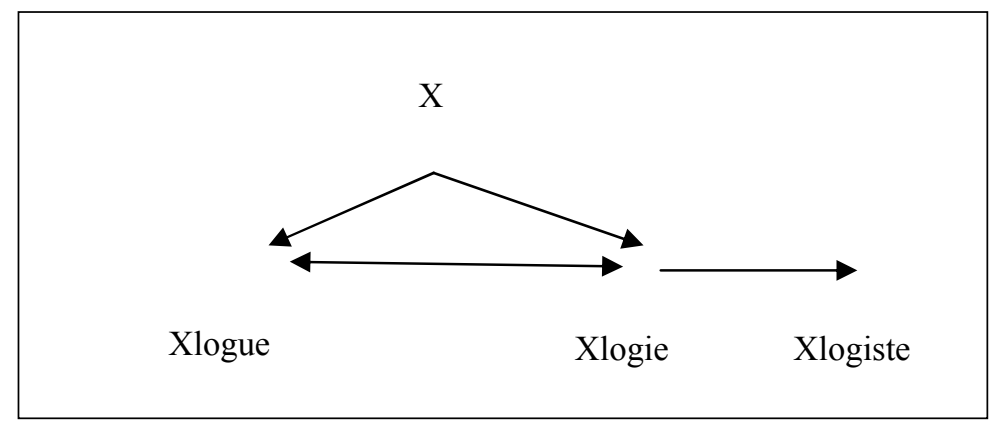

Figure 4.2 : Schéma (X, Xlogue, Xlogie, Xlogiste) 


\subsection{Relations paradigmatiques}

Nous venons de montrer que des lexèmes appartenant à des familles morphologiques distinctes, mettent en jeu des relations morphologiques similaires, schématiquement représentables sous forme de structures ternaires. En effet, des noms de la forme Xianisme, Xicien, Xisation et Xlogiste ont plusieurs bases constructionnelles possibles et sont souvent en relation morphologique biorientée avec l'une d'elles, ce qui fait que leur analyse est difficilement réalisable au moyen de règles unidirectionnelles binaires. $\mathrm{Au}$ contraire, ces noms entretiennent avec les éléments de leur famille morphologique des relations de nature paradigmatique.

Ce constat a été formulé dans la littérature de diverses manières et pour rentre compte de constructions morphologiques variées dans différentes langues.

Dans le cadre de la morphologie morphématique classique, par exemple, et suivant Van Marle (1985), Becker (1993) argumente, en faisant appel à l'analogie, en faveur de la nature fondamentalement paradigmatique de la morphologie lexicale en prenant pour exemples des cas de dérivations régressives et croisées. Camus et al. (1996) applique les mêmes principes pour rendre compte des dérivations en -ata en espagnol; Vallès (2003) analyse les constructions du catalan dans l'approche linguistique basée sur l'usage inspirée de Bybee (1985). Suivant une approche à base lexème, Plag (1999), Haspelmath (2002), puis Roché (2011) analysent les relations bi-orientées entre les noms en -ism(e) et ceux en -ist(e) au moyen de faisceaux de contraintes.

Enfin, G. Booij est le seul, à notre connaissance, à proposer une analyse unifiée de divers cas de schémas ternaires, et se sert pour cela des dispositifs offerts par le modèle de la morphologie des constructions qu'il a développé (voir notamment Booij, 2010). Il applique ce modèle à l'analyse de différentes constructions en néerlandais, comme par exemple la formation des noms d'agents déverbaux maculins et féminins, ou celle des gentilés (masculin et féminin) et des adjectifs ethniques, à partir du toponyme correspondant. L'analyse que nous proposons de nos données, si elle est actuellement schématisée au moyen de représentations graphiques, pourrait facilement se traduire sous forme de constructions et sousconstructions paradigmatiques dans ce cadre théorique.

\section{Conclusion}

À la suite de Roché (2009), notre attention a été attirée par l'existence de series d'unités lexicales complexes régulières présentant des suites de composants morphologiques, les formes en -ianisme, -icien, -logiste et -isation. Ces séquences, potentiellement analysables comme des suites affixales se sont révélées plus complexes qu'une simple concaténation de deux suffixes.

L'existence de ces séries entraîne l'émergence d'un nouveau modèle, non plus linéaire et orienté, mais ternaire, où chacune des trois formes constituant la famille des séries étudiées est en relation morphologique avec les deux autres. Si dans certains cas, les relations entre les formes sont nécessairement orientées, dans d'autres, le lien morphologique unissant deux des formes en présence est réversible. Ce constat met à mal certaines analyses traditionnelles en morphologie, qui requièrent nécessairement la succession d'opérations morphologiques orientées dans une structure forcément binaire. Enfin, notre représentation, où Xicien, Xianisme, Xisation et Xlogiste sont toujours reliables directement à $\mathrm{X}$, permet de rendre compte du fait que les séquences -icien,-ianisme, -isation et -logiste peuvent être perçues par les scripteurs comme des affixes à part entière.

L'analyse des données nous a par ailleurs permis de constater que ces séries étaient imputables à différentes motivations : d'ordre phonologique pour Xianisme et Xcien, sémantique pour Xlogiste et discursif pour Xisation. L'influence des contraintes d'ordre phonologique se manifeste par une substitution affixale pour la séquence -icien et par une substitution paradigmatique pour la séquence -ianisme. L'identité des contenus sémantiques de Xisation et Xiser explique leur interdépendance. À l'inverse, la relation sémantique entre Xlogue et Xlogiste impose de complexifier la représentation ternaire commune, pour rendre compte de leur non synonymie. 
Reste à observer d'autres cas, apparemment similaires, de séquences agglutinées de composants morphologiques pouvant être perçues comme autonomes, afin de vérifier si elles sont analysables au moyen du même dispositif, comme par exemple les formes en -isant (eg. latinisant, arabisant) ou en -aliser (eg. accidentaliser, mensualiser).

\section{Références bibliographiques}

Amiot D. \& Dal G. (2007). Integrating Combining Forms into a Lexeme-Based Morphology. In : G. Booij, B. Fradin, A. Ralli \& S. Scalise (eds), Online Proceedings of the Fifth Mediterranean Morphology Meeting (MMM5), Fréjus, 323-336.

Boyé, G. \& Plénat, M. (à paraître). L'allomorphie radicale dans les lexèmes adjectivaux du français. Le cas des adverbes en -ment. In : B. Tranel (ed), Understanding Allomorphy. Perspectives from Optimality Theory, Londres : Equinox Publishing.

Becker, T. (1993). Back-formation, cross-formation, and 'bracketing paradoxes' in paradigmatic morphology. Yearbook of Morphology 1992, 1-27.

Baroni, M. \& Bernardini, S. (eds.) (2006), Wacky! Working papers on the Web as Corpus, Bologna: GEDIT.

Baroni M., Bernardini S., Ferraresi A. \& Zanchetta E. (2009). The WaCky Wide Web: A Collection of Very Large Linguistically Processed Web-Crawled Corpora. Language Resources and Evaluation 43(3), 209-226.

Biber, D. (1986). Variation across Speech and Writing. Cambridge: Cambridge University Press.

Bonami, O., Boyé, G., \& Kerleroux, F. (2009). L'allomorphie radicale et la relation flexion-construction. In : B. Fradin, F. Kerleroux \& M. Plénat (éds), Aperçus de Morphologie du français, Paris : Presses Universitaires de Vincennes, 103-126.

Booij, G. (2010). Construction Morphology. Oxford: Oxford University Press.

Bybee, J. (1985). Morphology: A study of the relation between meaning and form. Philadelphia: Benjamins.

Camus Bergareche, B. \& Miranda Poza, A. (1996). En favor de una morfología paradigmática: las formaciones españolas en -ata. Revista Española de Lingüística 26(2) : 271-300.

Cruse D.A. (2004). Meaning in Language. Oxford: OUP.

Dal, G. (2004). Vers une morphologie de l'évidence : d'une morphologie de l'input à une morphologie de l'output, Mémoire d'habilitation à diriger des recherches, Université Lille 3.

Dal, G. \& Namer, F. (2010). Les noms en -ancelence du français : quel(s) patron(s) constructionnel(s) ? 2ème Congrès Mondial de Linguistique Française, New Orleans : ILF, 893-907.

Dal, G., \& Namer, F. (à paraître). La fréquence en morphologie : pour quels usages ?. Langages.

Dell, F. \& Selkirk, E. (1978). On a Morphologically Governed Vowel Alternation in French. In : S. J. Keyser (ed), Recent Transformational Studies in European Language, Cambridge: MIT Press, 1-51.

Fradin, B. (2009). IE, Romance: French. In : R. Lieber \& P. Štekauer (eds), The Oxford Handbook of Compounding, Oxford: Oxford University Press, 417-435.

Grammont, M. (1895). La dissimilation consonantique dans les langues indo-européennes et dans les langues romanes. Dijon : Imprimerie Danrantière.

Haspelmath, M. (2002). Understanding Morphology. London: Arnold.

Hathout, N. (2011). Une approche topologique de la construction des mots : propositions théoriques et application à la préfixation en anti-. In : M. Roché, G. Boyé, N. Hathout, S. Lignon \& M. Plénat (éds), Unités Morphologiques Au Lexique, London : Hermès Sciences Publishing, 251-318.

Koehl, A. (2009). Are French -ité Suffixed Nouns Property Nouns?. In : F. Montermini \& G. Boyé (eds), Morphology in Bordeaux - Selected Proceedings of Decembrettes 6.. Somerville, MA: Cascadilla Proceedings Project, 95-110. 
Lignon, S. (1999). Suffixasser ou suffixouiller?. In : D. Corbin, G. Dal, B. Fradin, B. Habert, F. Kerleroux, M. Plénat \& M. Roché (éds), La Morphologie des dérivés évaluatifs. Actes de Silexicales 2, Toulouse: Presses Universitaires de Lille, 1999, 117-126.

Lignon, S. (2000). La suffixation en -ien. Aspects sémantiques et phonologiques, Thèse de doctorat, Université Toulouse-Le Mirail.

Lignon, S. \& Plénat, M. (2009). Échangisme suffixal et contraintes phonologiques. In : B. Fradin, F. Kerleroux \& M. Plénat (éds), Aperçus de morphologie du français. Paris : Presses Universitaires de Vincennes, 65-81.

Lignon S. (2013). -iser and -ifier suffixations in French: Verify data to verize hypotheses?. In : N. Hathout, F. Montermini \& J. Tseng (eds), Selected Proceedings of the 7th Décembrettes: Morphology in Toulouse. München: Lincom Europa.

Mc Carthy J. \& Prince, A. (1993). Prosodic Morphology I : Constraint interaction and satisfaction. Ms., University of Massachusetts: Amherst \& Rutgers University.

Namer, F. (2013). Adjectival bases of French -aliser and -ariser verbs: syncretism or under-specification?. In : N. Hathout, F. Montermini \& J. Tseng (eds), Selected Proceedings of the 7th Décembrettes: Morphology in Toulouse. München: Lincom Europa, 185-210.

Plag, I. (1999). Morphological productivity. Structural constraints in English derivation. Berlin/New York: Mouton de Gruyter.

Plag I. (2003). Word-formation in English. Cambridge: Cambridge University Press.

Plénat, M. (1996a). De l'interaction des contraintes : une étude de cas. In : J. Durand \& B. Laks (eds), Current Trends in Phonology: Models and Methods, vol. 2, Salford : ESRI, University of Salford, 585-615.

Plénat, M. (1996b). Mots possibles et mots existants. Le cas des dérivés en -esque. In : G. Pérennou (éd.), Lexique et communication parlée, Séminaire GDR-PRC "Communication Homme-Machine", Toulouse : IRIT, Université Paul Sabatier, 113-125.

Plénat, M. (1996c). La suffixation en -Vche : données et problèmes. In : D. Genthial \& alii (éds), Séminaire Lexique. Représentations et Outils pour les Bases Lexicales, Morphologie Robuste, Grenoble : CLIPS-IMAG, Université de Grenoble, 53-62.

Plénat, M. (1997a). Morphophonologie des dérivés en -Vche. Recherches linguistiques de Vincennes 26, 113-150.

Plénat, M. (1997b). Analyse morphophonologique d'un corpus d'adjectifs dérivés en -esque. Journal of French Language Studies 7, 163-179.

Plénat, M. (1999). Distribution des suffixes évaluatifs verbaux en français. In : D. Corbin, G. Dal, B. Fradin, B. Habert, F. Kerleroux, M. Plénat \& M. Roché (éds), Les dérivés évaluatifs. Actes de Silexicales 2, Toulouse, Presses Universitaires de Lille, 179-188.

Plénat, M. (2000). Quelques thèmes de recherche actuels en morphophonologie française. Cahiers de lexicologie 77, $27-62$.

Plénat, M. \& Roché, M. (2004). Entre morphologie et phonologie : la suffixation décalée. Lexique 16, 159-198.

Plénat, M. (2005). Rosinette, cousinette, starlinette, chipinette. Décalage, infixation et épenthèse devant -ette. In : I. Choï-Jonin, M. Bras, A. Dagnac \& M. Rouquier (eds), Questions de classification en linguistique : méthodes et descriptions. Mélanges offerts au Professeur Christian Molinier. Berne : Peter Lang, 275-298.

Plénat M. (2011). Enquête sur les divers effets des contraintes dissimilatives en français. In : M. Roché, G. Boyé, N. Hathout, S. Lignon \& M. Plénat (éds), Des Unités Morphologiques Au Lexique, London : Hermès Sciences Publishing, 145-190.

Plénat, M. \& Roché, M. (2012). Éléments de morphologie quotidienne. Contribution à l'étude des conditionnements de l'épenthèse présuffixale. Congrès Mondial de Linguistique Française, Lyon : ILF.

Prince A. \& Smolensky, P. (1993). Optimality Theory: Constraint interaction in generative grammar. Ms., Rutgers University \& University of Colorado at Boulder. 
Roché M. (1997). Briard, bougeoir et camionneur : dérivés aberrants, dérivés possibles. In : D. Corbin, B. Fradin, B. Habert, F. Kerleroux \& M. Plénat (éds), Mots possibles et mots existants, Actes de Silexicales 1, Villeneuve d'Ascq : Presses de l’Université de Lille, 241-250.

Roché, M. (2002). La suffixation décalée avec oc. -ièr(a). In : L. Rabassa (ed), Mélanges offerts à Jean-Louis Fossat, Cahiers d'Etudes Romanes 11-12, 319-334.

Roché, M. (2003a), De la « bonne formation » des dérivés en -on. Cahiers de Grammaire 28, 91-112.

Roché, M. (2003b). L'interfixe est-il une unité morphologique ?. In : B. Fradin, G. Dal, N. Hathout, F. Kerleroux, M. Plénat \& M. Roché (éds) Les unités morphologiques, Actes de Silexicales 3, Villeneuve d'Ascq: Presses Universitaires du Septentrion, 169-178.

Roché, M. (2005). Interfixe et suffixation décalée dans les langues romanes. In : T. Arnavielle (ed), Langues : histoires et usages dans l'aire méditerranéenne, Paris : L'Harmattan, 71-87.

Roché, M. (2006). Comment les adjectifs sont sémantiquement construits. Cahiers de Grammaire 30, 373-387.

Roché M. (2007). Logique lexicale et morphologie : la dérivation en -isme. In : F. Montermini, G. Boyé \& N. Hathout (eds), Selected Proceedings of the 5th Décembrettes: Morphology in Toulouse. Sommerville, Mass.: Cascadilla Press, 1-51.

Roché, M. (2008). Structuration du lexique et principe d'économie : le cas des ethniques. In : J. Durand, B. Habert \& B. Laks (éds), Congrès Mondial de Linguistique Française - CMLF'08, Paris : ILF, 1571-1585.

Roché, M. (2009). Un ou deux suffixes? Une ou deux suffixations? In : B. Fradin, F. Kerleroux \& M. Plénat (eds), Aperçus de Morphologie du français.. Paris : Presses Universitaires de Vincennes, 143-174.

Roché, M. (2011a). Pression lexicale et contraintes phonologiques dans la dérivation en -aie du français, Linguistica $51,5-22$.

Roché M. (2011b). Quel traitement unifié pour les dérivations en -isme et en -iste ?, In : M. Roché, G. Boyé, N. Hathout, S. Lignon \& M. Plénat (eds), London : Hermès Sciences Publishing, Des unités morphologiques au lexique, 69-143.

Roché, M. \& Plénat, M. (2003). Prosodic constraints on suffixation in French. In : G. Booij, J. DeCesaris, A. Ralli \& S. Scalise (eds), Topics in Morphology. Selected Papers from the Third Mediterranean Morphology Meeting (Barcelona, September 20-22, 2001), Barcelona: I.U.L.A. Universitat Pompeu Fabra, 285-299.

Tanguy, L. (2013). La ruée linguistique vers le Web (URL : http://www.revue-texto.net/index.php?id=3335.). Texto XVIII -4.

Vallès, T. (2003). Lexical creativity and the organization of the lexicon. Annual Review of Cognitive Linguistics 1: 137-160.

van Marle, J. (1985). On the Paradigmatic Dimension of Morphological Creativity. Dordrecht: Foris Publications.

Villoing F. (2012). French compounds. Probus, 24 (1), 29-60.

Villoing, F. \& Namer, F. (à paraître). Composition néoclassique en -logue et -logiste: les noms en -logue sont-ils encore des noms de spécialistes ?. In : S. Lignon et F. Namer (eds), La composition néoclassique, Verbum, Nancy : Presses Universitaires de Nancy.

${ }^{1}$ Trésor de la Langue Française (1971-1994). Dictionnaire de la langue du XIXe et du XXe siècle, 16 volumes, CNRS, Gallimard, Paris. (TLFi : http://atilf.atilf.fr/tlf.htm et CDROM : CNRS édition, 2004.)

${ }^{2}$ Le TLF donne également la forme essenianisme comme construite sur un dérivé en -ien alors même que la base essenien n'est pas morphologiquement construite en français.

${ }^{3}$ Sauf mention contraire, les exemples donnés en contexte ont été trouvé sur la Toile courant 2013.

${ }^{4}$ Si l'on trouve quelques occurrences de la forme marialisme, elles sont très largement minoritaires par rapport à marinianisme.

${ }^{5}$ On trouve une occurrence de geekique mais cette forme est utilisée comme nom de maladie : «la geekique est la seule maladie qui se soigne avec des virus ». 


\footnotetext{
${ }^{6}$ Voir (Fradin, 2009 ; Villoing 2012) surt un panorama de la composition en français, et (Amiot \& Dal, 2007) pour une discussion sur le statut de-logue.

${ }^{7}$ Outil de mesure lexicométrique interrogeable en ligne et permettant de comparer les fréquences d'utilisation de (séquences de) termes à travers les publications recensées par Google. Ces résultats, qui dépendent de la quantité et du type d'ouvrages indexés dans Google Books, sont bien évidemment imparfaits, et ne doivent être interprétés que comme des tendances.

${ }^{8}$ Corpus du français de 1,6 milliards d'occurrences (Baroni \& al 2009) qui vise à reproduire, dans un format exploitable par des outils d'analyse automatique de corpus, la diversité des contenus récents de la Toile dans des proportions comparables à l'original. Ce corpus est le fruit du programme WaCKy de création d'outils et ressources pour l'anglais, l'allemand, l'italien et le français (Baroni et al 2006).

${ }^{9}$ Bien que les verbes apparentés soient absents du frWaC, on trouve sur la Toile 150 occurrrences de grégariser (356 grégarisation) et 41 cabaniser (251 cabanisation).

${ }^{10}$ Les requêtes ont porté sur les formes à l'infinitif

${ }^{11}$ Les requêtes ont porté sur les formes en -ise, -isé,-isée, -isées, -isés et -iser.
} 